\title{
Knockdown of long non-coding RNA PVT1 reverses multidrug resistance in colorectal cancer cells
}

\author{
HENG FAN $^{1-3^{*}}$, JIAN-HUA ZHU ${ }^{3 *}$ and XUE-QING YAO ${ }^{1,2}$ \\ ${ }^{1}$ The Second School of Clinical Medicine, Southern Medical University, Guangzhou, Guangdong 510515; \\ ${ }^{2}$ Department of General Surgery, Guangdong General Hospital and Guangdong Academy of \\ Medical Sciences, Guangzhou, Guangdong 510080; ${ }^{3}$ Department of Intensive Care Unit, \\ Ningbo First Hospital, Ningbo, Zhejiang 315000, P.R. China
}

Received December 25, 2017; Accepted March 22, 2018

DOI: $10.3892 / \mathrm{mmr} .2018 .8907$

\begin{abstract}
Multidrug resistance (MDR) is one of the primary causes of chemotherapy failure in colorectal cancer (CRC), and extensive biological studies into MDR are required. The non-coding RNA plasmacytoma variant translocation 1 (PVT1) has been demonstrated to be associated with low survival rates in patients with CRC. However, whether PVT1 serves a critical function in the MDR of CRC remains to be determined. To determine the association between PVT1 expression and 5-fluorouracil (5-FU) resistance in CRC, the expression levels of PVT1 mRNA in 5-FU-resistant CRC tissues and cell lines (HCT-8/5-FU and HCT-116/5-FU) were assessed by a reverse transcription-quantitative polymerase chain reaction (RT-qPCR). Cytotoxicity was evaluated using a Cell Counting Kit-8 assay and apoptosis rates were assessed via flow cytometry. In the present study, PVT1 mRNA was highly expressed in 5-FU-resistant CRC tissues and cell lines. HCT-8/5-FU and HCT-116/5-FU cells transfected with small interfering RNA PVT1 and treated with 5-FU exhibited higher apoptotic rates and lower survival rates. By contrast, overexpression of PVT1 in HCT- 8 and HCT-116 cells transfected with lentiviral vector-PVT1-green fluorescent protein and treated with 5-FU exhibited lower apoptosis rates and higher survival rates. RT-qPCR and western blotting demonstrated that the overexpression of PVT1 increased the mRNA and protein expression levels of multidrug resistance-associated protein 1, P-glycoprotein, serine/threonine-protein kinase mTOR and apoptosis regulator $\mathrm{Bcl} 2$. The present study indicates that PVT1 overexpression may promote MDR in CRC
\end{abstract}

Correspondence to: Professor Xue-Qing Yao, The Second School of Clinical Medicine, Southern Medical University, 1023 South Shatai Road, Baiyun, Guangzhou, Guangdong 510515, P.R. China E-mail: syyaoxueqing@scut.edu.cn

${ }^{*}$ Contributed equally

Key words: colorectal cancer, long non-coding RNA, PVT1, multidrug resistance cells, and suggested that inhibition of PVT1 expression may be an effective therapeutic strategy for reversing MDR in CRC.

\section{Introduction}

Colorectal cancer (CRC) is the third most common cancer type worldwide, and the fourth in China $(1,2)$. Chemotherapy has been the primary treatment for CRC in the past few decades, although multidrug resistance (MDR) frequently leads to its failure. Rebucci et al (3) reported a number of mechanisms of drug resistance, including alterations in drug metabolism, failure of DNA repair and apoptosis. Although these mechanisms have been demonstrated to be associated with the development of MDR in CRC, the regulators of chemotherapy resistance remain to be identified. Therefore, novel targeted approaches are required to clarify the underlying mechanisms of chemotherapy resistance, and more efficient therapies are required to counter MDR.

Long non-coding RNA plasmacytoma variant translocation 1 (lnc-PVT1) is located at 8q24 on the human chromosome (4), and a number of studies have reported that high expression of PVT1 reduces apoptosis in hepatocellular carcinoma (5), ovarian cancer (6) and breast cancer (7). Xu et al (8) identified that the expression of PVT1 is associated with the short and long-term prognosis of patients with gastric cancer, and that downregulation of PVT1 may be regarded as a therapeutic approach for gastric cancer. In addition, Fang et al (9) demonstrated that PVT1 is highly expressed in pancreatic cancer cells, and that sensitivity to chemotherapy is regulated by PVT1. However, whether PVT1 serves a critical function in the MDR of CRC is unclear.

The present study aimed to investigate the role of PVT1 in 5-fluorouracil (5-FU)-resistant CRC tissues and cell lines, and to further study the association between PVT1 expression and MDR-associated proteins, including MDR protein 1 (MRP1), P-glycoprotein (P-gp), serine/threonine-protein kinase mTOR (mTOR) and apoptosis regulator $\mathrm{Bcl} 2(\mathrm{Bcl}-2)$. The results in the present study may contribute to a novel therapeutic target for MDR in patients with CRC. 


\section{Materials and methods}

Patients and specimens. Samples of human tumor tissues were collected from 30 patients with primary CRC between September 2016 and December 2017. The patients with CRC (13 male and 17 female; $35-76$ years old with a median of 57.4 years) received 5-FU-based neoadjuvant chemotherapy prior to surgical removal of tumors. 5-FU-sensitive cases were defined by the following: Shrinkage of the primary tumor; no enlargement of the primary tumor; and no new occurrence of metastasis within 6-12 months $(n=15)$; otherwise, the cases were defined as 5-FU resistant $(n=15)$. The present study was approved by the Ethics Committees of Ningbo First Hospital (Zhejiang, China) and Guangdong General Hospital (Guangdong, China), and written informed consent was obtained from each patient.

Cells. CRC HCT-8 and HCT-116 cell lines (American Type Culture Collection, Manassas, VA, USA) were cultured, and 5-FU (Sigma-Aldrich; Merck KGaA, Darmstadt, Germany) was used to generate drug-resistant CRC cells. HCT- 8 and HCT-116 cells were exposed to 5-FU in vitro at a continual stepwise increasing dose $(5,7.5,10$ and $20 \mu \mathrm{M})(10)$. All CRC cells were cultured in RPMI-1,640 medium (Thermo Fisher Scientific, Inc., Waltham, MA, USA) with $10 \%$ fetal bovine serum (Sigma-Aldrich; Merck KGaA, Darmstadt, Germany) at $37^{\circ} \mathrm{C}$ with $5 \% \mathrm{CO}_{2}$.

Cell transfection and cytotoxicity assay. The lentiviral transfection vector of PVT1 overexpression tagged with green fluorescent protein (LV-PVT1-GFP) and the negative control (NC) lentiviral vector were purchased from the Shanghai Tumor Research Institute (Shanghai, China). Transfection of PVT1 small interfering (si)RNA (Guangzhou RiboBio Co., Ltd., Guangzhou, China) was performed with Lipofectamine ${ }^{\circledR}$ 2000 (Invitrogen; Thermo Fisher Scientific, Inc.), according to the manufacturer's protocol. PVT1 siRNA sense 5'-CGA ACCTCCGUCCTCCCTATT-3' and antisense 5'-TTGAGG AGTCGGAUGTCT-3' strands were used. The lentiviral vector that carried a non-targeting sequence was used as the $\mathrm{NC}$ of siPVT1. HCT-8/5-FU and HCT-116/5-FU cells were seeded into 6-well plates for $24 \mathrm{~h}$, and subsequently transfected with PVT1 siRNA oligonucleotides or a viral supernatant in Opti-minimum essential medium (Invitrogen; Thermo Fisher Scientific, Inc.); the transfected cells were grown at $37^{\circ} \mathrm{C}$ with $5 \% \mathrm{CO}_{2}$ for $24-48 \mathrm{~h}$, and stable cells were harvested for further study. A Cell Counting Kit-8 (CCK-8; Sigma-Aldrich; Merck $\mathrm{KGaA}$ ) assay was used to assess cell viability and survival rates, according to the manufacturer's protocol (11). CRC cells were incubated in 96-well plates at a density of $5 \times 10^{4}$ cells/well and were subsequently exposed to 4 different concentrations of 5-FU $(0.25,0.5,0.75$ and $1.0 \mu \mathrm{g} / \mathrm{ml})$. Following incubation, $10 \mu$ CCK-8 reagent was added and the cells were cultured for a further $2 \mathrm{~h}$ at $37^{\circ} \mathrm{C}$ and $5 \% \mathrm{CO}_{2}$. The absorbance was measured at $450 \mathrm{~nm}$ using a microplate reader (Xi'an Guanyu Bio-Tech Co., Ltd., Xi'an, China).

Measurement of apoptosis. The cells were trypsinized and fixed in $75 \%$ ethanol at $4^{\circ} \mathrm{C}$ for $25-30 \mathrm{~min}$. The cell pellets were subsequently harvested and incubated using an Annexin
V-fluorescein isothiocyanate/propidium iodide apoptosis detection kit (Invitrogen; Thermo Fisher Scientific, Inc.). Cellular apoptosis was evaluated via flow cytometry (Phoenix Flow Systems, San Diego, CA, USA) within 5 min and analyzed using a flow cytometer (Coulter EPICS XL-MCL FACScan, BD Biosciences, Franklin Lakes, NJ, USA). The data was analyzed using the MultiCycle Software for Windows version 5.0 (Phoenix Flow Systems, San Diego, CA, USA).

Western blotting. The cellular proteins were extracted and separated electrophoretically via SDS-PAGE, and subsequently probed with antibodies against MRP1 (cat. no. ab3368, 1:1,500; Abcam, Cambridge, UK), P-gp (cat. no. ab129450, 1:3,000; Santa Cruz Biotechnology, Inc., Dallas, TX, USA), mTOR (cat. no. ab2732, 1:1,500, Abcam), Bcl-2 (cat. no. ab59348, 1:1,000; Santa Cruz Biotechnology, Inc.), and anti-GAPDH (cat. no. ab9485, 1:1,000; Santa Cruz Biotechnology, Inc.) as a control. Quantification of the expression levels of these proteins was performed using Quantity-One-Software 29.0 (Bio-Rad Laboratories, Inc., Hercules, CA, USA).

Reverse transcription-quantitative polymerase chain reaction $(R T-q P C R)$. Total RNA was extracted from fresh frozen CRC tissues or cells using TRIzol ${ }^{\circledR}$ (Life Technologies; Thermo Fisher Scientific, Inc.), according to the manufacturer's protocol. cDNA was synthesized using the $\mathrm{Hi}-\mathrm{Fi}$ Script Reverse Transcriptase kit (CWBio, Beijing, China). Amplification and detection of mRNA was performed using SYBRGreen (Applied Biosystems; Thermo Fisher Scientific, Inc.), with GAPDH as the internal control. The primer sequences used were as follows: PVT1, forward 5'-CAGCAC TCTGGACGGAC-3'; reverse 5'-CAACAGGAGAAGCAA ACA-3'; MRP1, forward 5'-GCACGTGCACTACCATGT CA-3'; reverse 5'-CTGGTCTCTGCACTCATCTTGCGC-3'; P-gp, forward 5'-GCACGCATCGCCTTAG-3', reverse 5'-AGT TGTCCATCATTATCCC-3'; mTOR, forward 5'-GCCCAG ACTGCGATGCCAGTAGG-3', reverse 5'-GAGCACTGA CGACAGTACCAGGCC-3'; Bcl-2, forward 5'-AGCAGCAAG TAGGTGTCCCAG-3', reverse 5'-CTCCACGCCATCTTG CTTCT-3'; GAPDH, forward 5'-TCCAGAGTGCAAGGCTTC AG-3', reverse 5'-ACAGCACGCAGTAGCAGTA-3'. The PCR conditions were as follows: $94^{\circ} \mathrm{C}$ for $2 \mathrm{~min}$, followed by $94^{\circ} \mathrm{C}$ for $30 \mathrm{sec}, 60^{\circ} \mathrm{C}$ for $30 \mathrm{sec}$ and $72^{\circ} \mathrm{C}$ for $1 \mathrm{~min}$ for 30 cycles, and $72^{\circ} \mathrm{C}$ for $10 \mathrm{~min}$. The relative expression of mRNA levels was calculated using the $2^{-\Delta \Delta \mathrm{Cq}}$ method (12).

Statistical analysis. The data are presented as the mean \pm standard deviation. Statistical analysis and graph construction was conducted using GraphPad version 7.5 software (GraphPad Software, Inc., La Jolla, CA, USA). All experiments were repeated three times, and differences between the groups were analyzed using one-way analysis of variance with Fisher's least significance difference test as the post hoc test. $\mathrm{P}<0.05$ was considered to indicate a statistically significant difference.

\section{Results}

IncRNA PVT1 is associated with 5-FU resistance in human CRC tissues and cells. MDR is a key factor leading to failure of chemotherapy. A previous study indicated that overexpression 
A

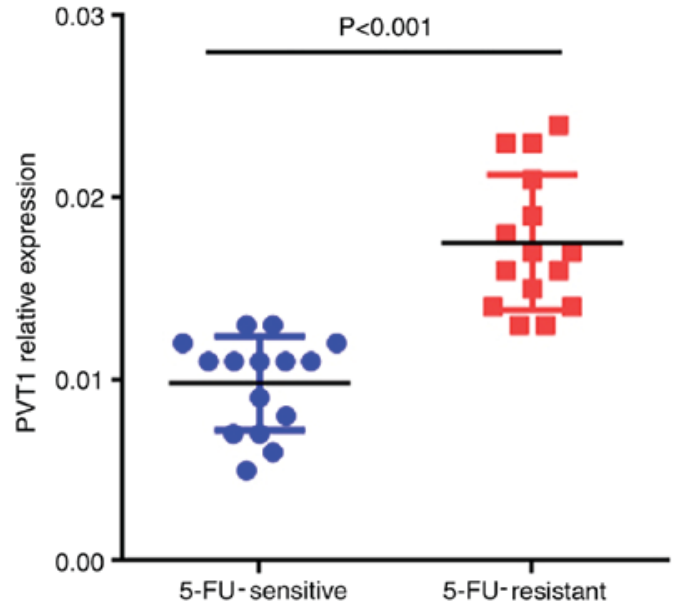

B

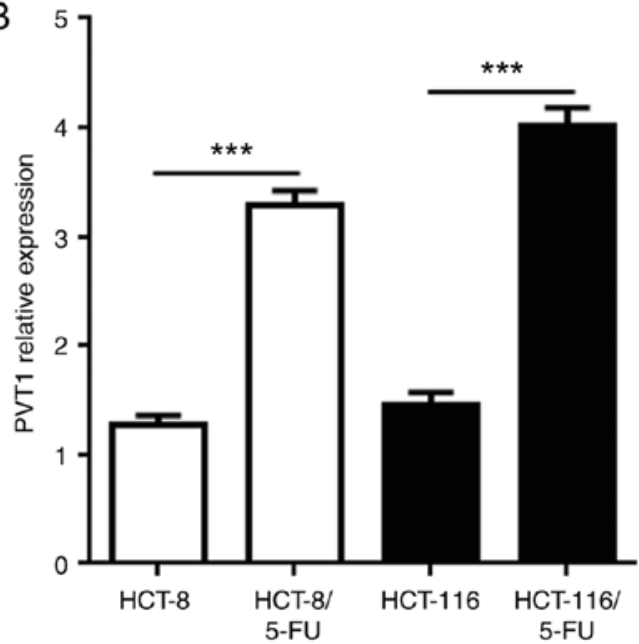

Figure 1. PVT1 is associated with 5-FU-resistance. (A) The expression levels of PVT1 mRNA in CRC tissues from 5-FU-sensitive and 5-FU-resistant patients. (B) The expression levels of PVT1 mRNA in 5-FU-resistant HCT-8 and 5-FU-resistant HCT-116 cells. All values are presented as the mean \pm standard deviation. ${ }^{* * *} \mathrm{P}<0.001$. PVT1, plasmacytoma variant translocation 1; 5-FU, 5-fluorouracil; CRC, colorectal cancer.

of PVT1 in human colonic mucosal cells is associated with increased morbidity from CRC (13). However, whether PVT1 is associated with MDR remains to be elucidated. To determine the association between PVT1 expression and 5-FU resistance, 5-FU-sensitive $(n=15)$ and 5-FU-resistant $(n=15)$ tissues were collected from patients with CRC to examine the expression of PVT1 mRNA. The results of the present study demonstrated that the PVT1 mRNA expression was significantly upregulated in 5-FU-resistant CRC tissues compared with that in the 5-FU-sensitive CRC tissues (Fig. 1A; $\mathrm{P}<0.001$ ). To further investigate these different expression levels of PVT1 mRNA, two 5-FU resistant human CRC cell lines (HCT-8/5-FU and HCT-116/5-FU) were established by exposing the cells to 5-FU. The results demonstrated that PVT1 mRNA exhibited significantly higher expression in HCT-8/5-FU and HCT-116/5-FU cells compared with the untreated cells of the same cell type (Fig. 1B; $\mathrm{P}<0.001$ ). These results suggested that the expression of PVT1 may be associated with the development of 5-FU resistance in human CRC.

Knockdown of IncRNA PVT1 reverses drug resistance in 5-FU-resistant CRC cells. To further investigate the potential associations between the expression levels of PVT1 and 5-FU resistance in CRC, PVT1 was knocked down in HCT-8/5-FU and HCT-116/5-FU cells, and cellular cytotoxicity and apoptosis were examined. The expression of PVT1 mRNA was significantly decreased in HCT-8/5-FU and HCT-116/5-FU cells exposed to siPVT1 compared with the NC of each respective cell line (Fig. 2A; $\mathrm{P}<0.001$ ). Following transfection with siPVT1, the two drug resistant cell lines were treated with 5-FU for 5 days (The median effective dose value was the smallest at $0.5 \mu \mathrm{g} / \mathrm{ml} 5-\mathrm{FU}$, thus $0.5 \mu \mathrm{g} / \mathrm{ml} 5-\mathrm{FU}$ was used in the experiment). The CCK- 8 assay demonstrated that the cells transfected with siPVT1 had decreased viability and survival rates compared with the NC groups of the same cell line at the same time points following treatment with 5-FU (Fig. 2B and C). To confirm whether the decreased viability and survival rates of these cells were induced by apoptosis, the apoptosis rate was determined following transfection with
siPVT1 and treatment with 5 -FU $(0.5 \mu \mathrm{g} / \mathrm{ml})$. The results of the present study demonstrated that the proportion of apoptotic cells was significantly increased in the siPVT1 groups of the two cell lines compared with the NC groups (Fig. 2D and E; $\mathrm{P}<0.01)$, suggesting that the knockdown of PVT1 may reverse the drug resistance of 5-FU-resistant CRC cell lines by inducing apoptosis.

Overexpression of IncRNA PVT1 suppresses the apoptosis of CRC cells. Following transfection with LV-PVT1-GFP and treatment with 5-FU, the association between PVT1 overexpression and cellular apoptosis was further evaluated. The PVT1 mRNA levels in LV-PVT1-GFP-transfected HCT-8 and HCT-116 cells were 7.6- and 7.2-fold higher, respectively, compared with cells in the NC groups (Fig. 3A). These results indicated that LV-PVT1-GFP-transfected HCT-8 and HCT-116 cells contributed to the upregulation of PVT1 mRNA levels. Furthermore, the CCK-8 assay demonstrated that LV-PVT1-GFP transfected HCT-8 and HCT-116 cells had higher viability compared with the respective $\mathrm{NC}$ groups (Fig. 3B and C), and the flow cytometric analysis indicated that LV-PVT1-GFP transfected HCT-8 and HCT-116 cells had a significantly decreased apoptosis rate compared with cells in the NC groups (Fig. 3D and E; P<0.01). These results indicated that PVT1 overexpression suppressed 5-FU induced apoptosis in HCT-8 and HCT-116 cells.

Association between lncRNA PVT1 and MDR-associated proteins. High expression of MDR-related proteins is considered to be one of the primary MDR mechanisms in tumor cells (14). To study the effect of PVT1 overexpression in 5-FU resistant CRC cell lines, the mRNA of MDR-associated proteins was detected by RT-qPCR, including MRP1, P-gp and mTOR and an inhibitor of apoptosis, Bcl-2. The mRNA expression levels of MRP1, P-gp, mTOR and Bcl-2 in LV-PVT1-GFP-transfected HCT-8 and HCT-116 cells were significantly increased compared with the cells of the NC groups (Fig. 4A and B; $\mathrm{P}<0.001$ ). In addition, the protein expression levels of MRP1, P-gp, mTOR and Bcl-2 were examined 

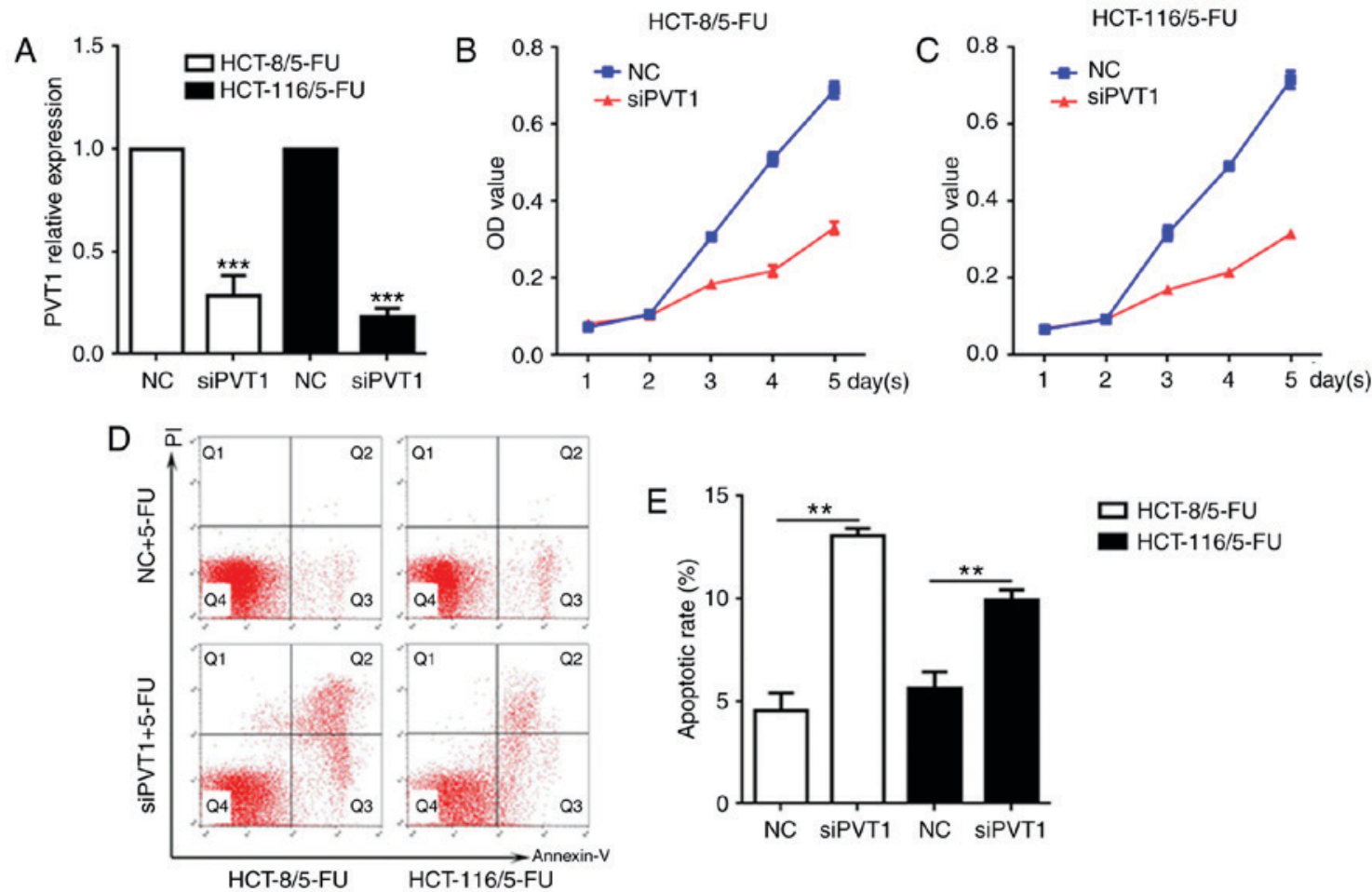

Figure 2. Knockdown of PVT1 reverses drug resistance in 5-FU-resistant CRC cells. (A) PVT1 mRNA levels in HCT-8/5-FU and HCT-116/5-FU following silencing of PVT1. (B) The effect of PVT1 knockdown on the viability of HCT-8/5-FU cells transfected with siPVT1 and treated with 5-FU. (C) The effect of PVT1 knockdown on the viability of HCT-116/5-FU cells transfected with siPVT1 and treated with 5-FU. The proportion of apoptotic cells in HCT-8/5-FU cells HCT-116/5-FU cells transfected with siPVT1 was determined by (D) flow cytometry and (E) quantified. All values are presented as the mean \pm standard deviation. ${ }^{* *} \mathrm{P}<0.01$ and ${ }^{* * *} \mathrm{P}<0.001$ vs. respective NC. PVT1, plasmacytoma variant translocation 1; 5-FU, 5-fluorouracil; CRC, colorectal cancer; OD, optical density, PI, propidium iodide; NC, negative control; si, small interfering.
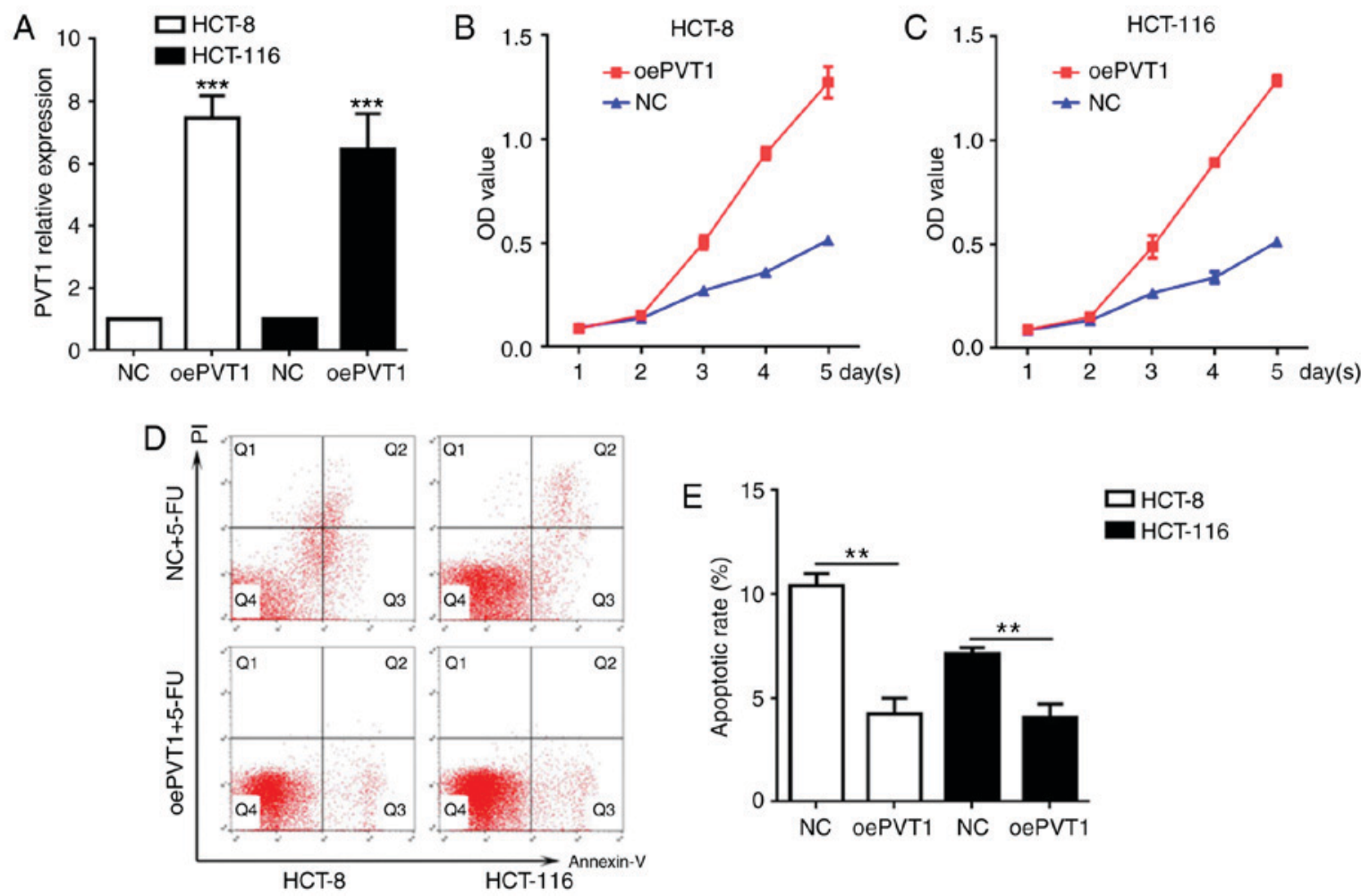

Figure 3. Overexpression of PVT1 suppresses apoptosis in CRC cells. (A) LV-PVT1-GFP-transfected cells overexpressed PVT1. (B) The effect of PVT1 overexpression on the viability of HCT-8 cells transfected with LV-PVT1-GFP and treated with 5-FU. (C) The effect of PVT1 overexpression on the cell viability of HCT-116 cells transfected with LV-PVT1-GFP and treated with 5-FU. The percentage of apoptotic cells in HCT-8/5-FU cells and HCT-116/5-FU cells transfected with LV-PVT1-GFP was analyzed by (D) flow cytometry and (E) quantified. All values are presented as the mean \pm standard deviation. ${ }^{* *} \mathrm{P}<0.01$ and ${ }^{* * *} \mathrm{P}<0.001$ vs. respective NC. PVT1, plasmacytoma variant translocation 1; CRC, colorectal cancer; 5-FU, 5-fluorouracil; LV-PVT1-GFP, lentiviral vector-PVT1-green fluorescent protein; PI, propidium iodide; OD, optical density; oePVT1, overexpression of PVT1; NC, negative control. 

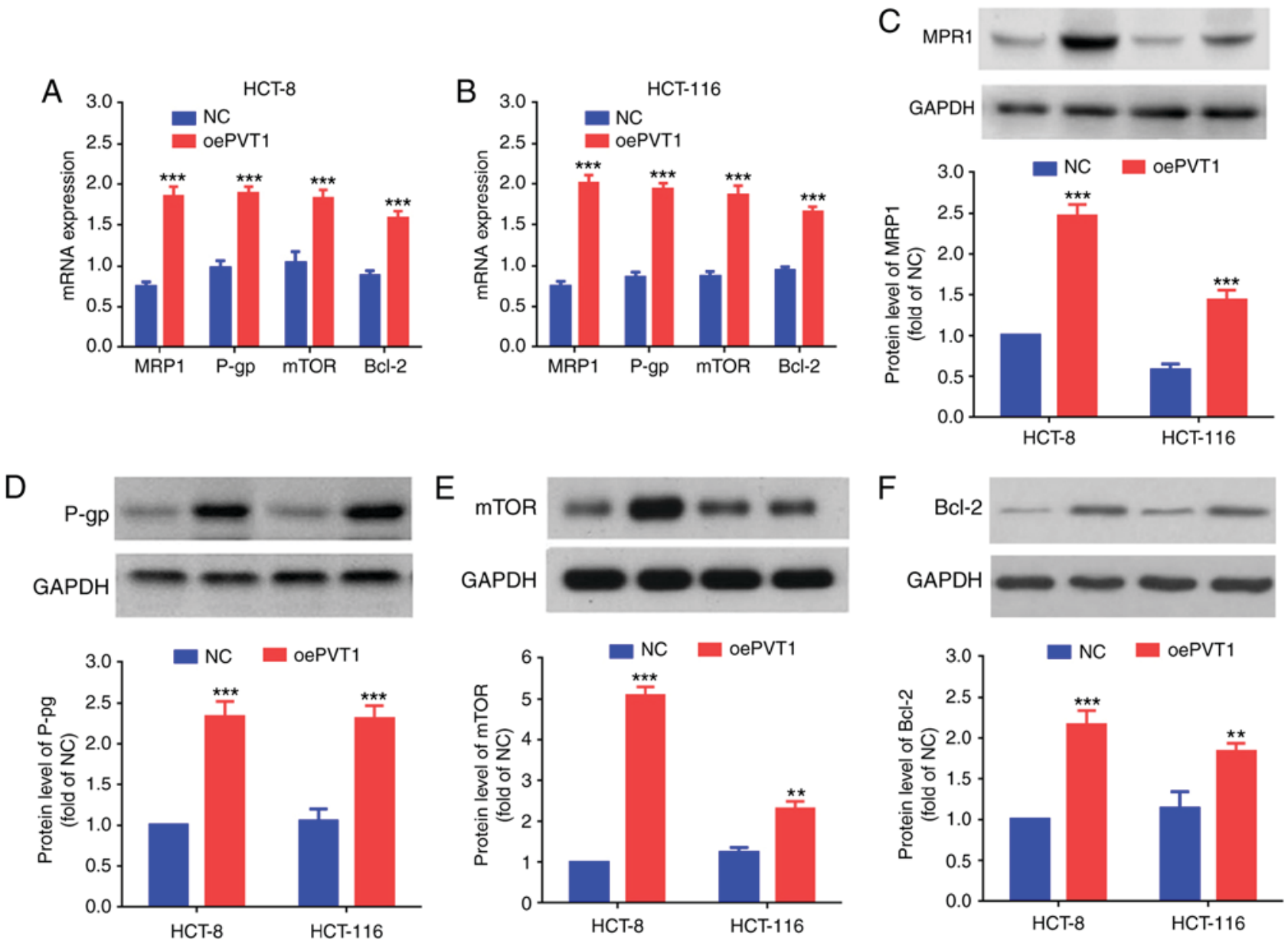

Figure 4. PVT1 influences the expression of genes and proteins associated with MDR. mRNA expression levels of MRP1, P-gp, mTOR and Bcl-2 in (A) the HCT-8 cells transfected with LV-PVT1-GFP and (B) the HCT-116 cells transfected with LV-PVT1-GFP. Protein expression levels of (C) MRP1, (D) P-gp, (E) mTOR and (F) Bcl-2 in the HCT-8/5-FU cells and HCT-116/5-FU cells transfected with LV-PVT1-GFP. GAPDH was used as a loading control. All values are presented as the mean \pm standard deviation. ${ }^{* *} \mathrm{P}<0.01$ and ${ }^{* * * *} \mathrm{P}<0.001$ vs. respective NC. PVT1, plasmacytoma variant translocation $1 ; 5-\mathrm{FU}, 5$-fluorouracil; LV-PVT1-GFP, lentiviral vector-PVT1-green fluorescent protein; MDR, multidrug resistance; NC, negative control; MRP1, multidrug resistance protein 1; P-gp, P-glycoprotein; mTOR, serine/threonine-protein kinase mTOR; Bcl-2, apoptosis regulator Bcl2; oePVT1, overexpression of PVT1.

via western blot analysis. The protein expression levels of MRP1, P-gp, mTOR and Bcl-2 were significantly increased in the LV-PVT1-GFP-transfected HCT-8 and HCT-116 cells, compared with the cells of the NC groups (Fig. 4C-F).

\section{Discussion}

Mortality from CRC has been reduced due to commitment to CRC screening, the reduction of risk factors, and improvements in therapeutic measures, although the morbidity in a number of countries continues to increase (15). 5-FU has been widely used as an important chemotherapeutic agent in CRC, as it is able to rapidly shrink tumor mass; however, MDR may be a cause of failure to eliminate tumor cells thoroughly. Therefore, it is necessary to clarify the exact mechanisms of MDR.

Numerous studies have elucidated the effectiveness of lncRNA PVT1 in human cancer, including breast (7), cervical (16), thyroid (17), gastric (18) and ovarian (19) cancer. In these previous studies, PVT1 has been demonstrated to exert different effects. Liu et al (6) demonstrated that the overexpression of PVT1 facilitates cellular apoptosis in ovarian cancer and inhibits tumor growth, suggesting that PVT1 has antitumor properties. By contrast, Wan et al (20) identified that PVT1 overexpression is associated with increased lung cancer lymph node metastasis and poor overall survival, while PVT1 knockdown may inhibit cell proliferation and induce apoptosis, indicating that PVT1 has potential oncogenic activity. In addition, certain studies have reported that PVT1 facilitates the development of MDR in certain cancer cases $(5,21)$. Zhang et al (21) suggested that PVT1 overexpression promotes the development of cisplatin-resistance in gastric carcinoma and that PVT1 may be a potential target for reversing MDR.

The results of the present study demonstrated that PVT1 was highly expressed in the CRC tissues of 5-FU resistant patients, and in HCT-8/5-FU and HCT-116/5-FU cells, and that PVT1 overexpression was associated with the development of 5-FU resistance. Previous studies have demonstrated that cellular apoptosis serves an important function in MDR, and the majority of chemotherapeutic drugs induce tumor cellular apoptosis to exert their anticancer activity (22-24). Therefore, inhibiting tumor cellular apoptosis is one of the principal mechanisms of MDR. Takahashi et al (25) reported that PVT1 had anti-apoptotic activity in CRC. However, no current study, to the best of the authors' knowledge, has demonstrated the 
exact role of PVT1 in the induction of apoptosis in CRC. In the present study, knockdown of PVT1 significantly inhibited viability and increased the apoptosis rate following transfection with siPVT1 and treatment with 5-FU. By contrast, PVT1 overexpression had the property of inhibiting apoptosis, and suppressed the apoptosis of HCT- 8 and HCT-116 cells treated with 5-FU. These results suggested that siPVT1 may reverse the 5-FU resistance of 5-FU resistant cells.

MRP1 is a member of the adenosine triphosphate-binding cassette superfamily of transmembrane transporters, and, via overexpression of P-gp, causes MDR (26). P-gp is regarded as a transmembrane efflux pump, which prevents the accumulation of chemotherapeutic drugs (27). The expression level of MRP1/P-gp is regulated by various signaling pathways and may affect the therapeutic efficacy of chemotherapeutic agents (28). MRP1, P-gp and mTOR are regarded as important drug resistance molecular targets in cancer therapy. Yan et al (29) reported that MRP1 and mTOR contributed to the upregulation of P-gp, leading to inhibition of accumulation of chemotherapeutic drugs in cells. These proteins were investigated in the present study to clarify the critical role of PVT1 in MDR. The results indicated that PVT1 upregulated the expression of MRP1, P-gp, mTOR and Bcl-2, supporting the hypothesis that PVT1 may promote the development of MDR in CRC.

The present study demonstrated that PVT1 knockdown reverses drug resistance in 5-FU resistant CRC cell lines, and that PVT1 overexpression promotes the development of MDR in CRC primarily by inhibiting apoptosis and upregulating the expression of MRP1, P-gp, mTOR and Bcl-2. The results of the present study indicated that PVT1 is a potential therapeutic target for the treatment of MDR in CRC.

\section{Acknowledgements}

Not applicable.

\section{Funding}

The present study was supported by the Zhejiang Provincial Medical and Health Science Fund (grant no. 2018KY667).

\section{Availability of data and materials}

All data generated or analyzed during this study are included in this published article.

\section{Authors' contributions}

HF and JHZ performed the experiments and drafted the manuscript. XQY designed the experiments and revised the manuscript. All authors read and approved the final manuscript.

\section{Ethics approval and consent to participate}

The present study was approved by the Ethics Committee of Ningbo First Hospital (Zhejiang, China) and Guangdong General Hospital (Guangdong, China), and written informed consent was obtained from each patient.

\section{Consent for publication}

All patients provided written informed consent for the publication of this article.

\section{Competing interests}

The authors declare that they have no competing interests.

\section{References}

1. Ferlay J, Parkin DM and Steliarova-Foucher E: Estimates of cancer incidence and mortality in Europe in 2008. Eur J Cancer 46: 765-781, 2010.

2. Xu M, Chen YM, Huang J, Fang YJ, Huang WQ, Yan B, Lu MS, Pan ZZ and Zhang CX: Flavonoid intake from vegetables and fruits is inversely associated with colorectal cancer risk: A case-control study in China. Br J Nutr 116: 1275-1287, 2016.

3. Rebucci M and Michiels C: Molecular aspects of cancer cell resistance to chemotherapy. Biochem Pharmacol 85: 1219-1226, 2013.

4. Beck-Engeser GB, Lum AM, Huppi K, Caplen NJ, Wang BB and Wabl M: Pvt1-encoded microRNAs in oncogenesis. Retrovirology 5: 4, 2008.

5. Yang X, Xie X, Xiao YF, Xie R, Hu CJ, Tang B, Li BS and Yang SM: The emergence of long non-coding RNAs in the tumorigenesis of hepatocellular carcinoma. Cancer Lett 360: 119-124, 2015.

6. Liu E, Liu Z and Zhou Y: Carboplatin-docetaxel-induced activity against ovarian cancer is dependent on up-regulated lncRNA PVT1. Int J Clin Exp Pathol 8: 3803-3810, 2015.

7. Paci P, Colombo T and Farina L: Computational analysis identifies a sponge interaction network between long non-coding RNAs and messenger RNAs in human breast cancer. BMC Syst Biol 8: 83, 2014.

8. Xu MD, Wang Y, Weng W, Wei P, Qi P, Zhang Q, Tan C, Ni SJ, Dong L, Yang Y, et al: A positive feedback loop of lncRNA-PVT1 and FOXM1 facilitates gastric cancer growth and invasion. Clin Cancer Res 23: 2071-2080, 2017.

9. Fang XY, Pan HF, Leng RX and Ye DQ: Long noncoding RNAs: Novel insights into gastric cancer. Cancer Lett 356: 357-366, 2015.

10. Uchibori K, Kasamatsu A, Sunaga M, Yokota S, Sakurada T, Kobayashi E, Yoshikawa M, Uzawa K, Ueda S, Tanzawa H and Sato N: Establishment and characterization of two 5-fluorouracil-resistant hepatocellular carcinoma cell lines. Int J Oncol 40: 1005-1010, 2012.

11. Wang Y, Zhou J, Wang Z, Wang P and Li S: Upregulation of SOX2 activated LncRNA PVT1 expression promotes breast cancer cell growth and invasion. Biochem Biophys Res Commun 493: 429-436, 2017.

12. Livak KJ and Schmittgen TD: Analysis of relative gene expression data using real-time quantitative PCR and the 2(-Delta Delta C(T)) method. Methods 25: 402-408, 2001.

13. Farhana L, Antaki F, Anees MR, Nangia-Makker P, Judd S, Hadden T, Levi E, Murshed F, Yu Y, Van Buren E, et al: Role of cancer stem cells in racial disparity in colorectal cancer. Cancer Med 5: 1268-1278, 2016.

14. Wu Q, Yang Z, Nie Y, Shi Y and Fan D: Multi-drug resistance in cancer chemotherapeutics: Mechanisms and lab approaches. Cancer Lett 347: 159-166, 2014.

15. Torre LA, Bray F, Siegel RL, Ferlay J, Lortet-Tieulent J and Jemal A: Global cancer statistics, 2012. CA Cancer J Clin 65: 87-108, 2015.

16. Iden M, Fye S, Li K, Chowdhury T, Ramchandran R and Rader JS: The lncRNA PVT1 contributes to the cervical cancer phenotype and associates with poor patient prognosis. PLoS One 11: e0156274, 2016.

17. Zhou Q, Chen J, Feng J and Wang J: Long noncoding RNA PVT1 modulates thyroid cancer cell proliferation by recruiting EZH2 and regulating thyroid-stimulating hormone receptor (TSHR). Tumour Biol 37: 3105-3113, 2016.

18. Kong R, Zhang EB, Yin DD, You LH, Xu TP, Chen WM, Xia R, Wan L, Sun M, Wang ZX, et al: Long noncoding RNA PVT1 indicates a poor prognosis of gastric cancer and promotes cell proliferation through epigenetically regulating $\mathrm{p} 15$ and $\mathrm{p} 16 . \mathrm{Mol}$ Cancer 14: 82, 2015. 
19. Liu E, Liu Z, Zhou Y, Mi R and Wang D: Overexpression of long non-coding RNA PVT1 in ovarian cancer cells promotes cisplatin resistance by regulating apoptotic pathways. Int J Clin Exp Med 8: 20565-20572, 2015.

20. Wan L, Sun M, Liu GJ, Wei CC, Zhang EB, Kong R, Xu TP Huang MD and Wang ZX: Long non-coding RNA PVT1 promotes non-small cell lung cancer cell proliferation through epigenetically regulating LATS2 expression. Mol Cancer Ther 15: 1082-1094, 2016.

21. Zhang XW, Bu P, Liu L, Zhang XZ and Li J: Overexpression of long non-coding RNA PVT1 in gastric cancer cells promotes the development of multidrug resistance. Biochem Biophys Res Commun 462: 227-232, 2015.

22. Duarte N, Varga A, Cherepnev G, Radics R, Molnár J and Ferreira MJ: Apoptosis induction and modulation of P-glycoprotein mediated multidrug resistance by new macrocyclic lathyrane-type diterpenoids. Bioorg Med Chem 15: 546-554, 2007.

23. Wobst I, Ebert L, Birod K, Wegner MS, Hoffmann M, Thomas D, Angioni C, Parnham MJ, Steinhilber D, Tegeder I, et al: R-flurbiprofen traps prostaglandins within cells by inhibition of multidrug resistance-associated protein-4. Int J Mol Sci 18: pii: E68, 2016.

24. Wang MZ, Qiu CZ, Yu WS, Guo YT, Wang CX and Chen ZX: GOLPH3 expression promotes the resistance of HT29 cells to 5 -fluorouracil by activating multiple signaling pathways. Mol Med Rep 17: 542-548, 2018.
25. Takahashi Y, Sawada G, Kurashige J, Uchi R, Matsumura T, Ueo H, Takano Y, Eguchi H, Sudo T, Sugimachi K, et al: Amplification of PVT-1 is involved in poor prognosis via apoptosis inhibition in colorectal cancers. Br J Cancer 110: 164-171, 2014.

26. Weerasinghe P, Hallock S, Tang SC, Trump B and Liepins A: Sanguinarine overcomes p-glycoprotein-mediated multidrug-resistance via induction of apoptosis and oncosis in CEM-VLB 1,000 cells. Exp Toxicol Pathol 58: 21-30, 2006.

27. Xie Y, Yu N, Chen Y, Zhang K, Ma HY and Di Q: HMGB1 regulates P-glycoprotein expression in status epilepticus rat brains via the RAGE/NF- $\mathrm{RB}$ signaling pathway. Mol Med Rep 16: 1691-1700, 2017

28. Gottesman MM and Pastan IH: The role of multidrug resistance efflux pumps in cancer: Revisiting a JNCI publication exploring expression of the MDR1 (P-glycoprotein) gene. J Natl Cancer Inst 107: pii: djv222, 2015.

29. Yan LH, Wei WY, Cao WL, Zhang XS, Xie YB and Xiao Q: Overexpression of $\mathrm{CDX} 2$ in gastric cancer cells promotes the development of multidrug resistance. Am J Cancer Res 5: 321-332, 2014.

(i) (2) This work is licensed under a Creative Commons Attribution-NonCommercial-NoDerivatives 4.0 International (CC BY-NC-ND 4.0) License. 